\title{
Reabsorption Losses in Luminescent Solar Concentrators: Effect of the Band Gap of Semiconductor Quantum Dots, their Size and Dispersion
}

\author{
A.I. Shkrebtii ${ }^{1 *}$, A.V. Sachenko ${ }^{2}$, I.O. Sokolovskyi ${ }^{2}$, V.P. Kostylyov ${ }^{2}$, M.R. Kulish ${ }^{2}$, \\ and D.V. Khomenko ${ }^{2}$ \\ ${ }^{1}$ University of Ontario Institute of Technology, 2000 Simcoe St. N., Oshawa, Ontario, L1H 7K4, Canada \\ *Corresponding author. Phone number: +1-905-721-8668 ext.2558, e-mail: anatoli.chkrebtii@uoit.ca \\ ${ }^{2}$ V. Lashkaryov Institute of Semiconductor Physics, NAS of Ukraine, 45 Pr. Nauky, Kyiv 03028, Ukraine
}

\begin{abstract}
We investigate the effect of semiconductor quantum dots (QDs) radius $\bar{r}$ and its dispersion $\Delta \bar{r}$ on the reabsorption during a luminescence process. QDs are promising as chromophores in luminescence solar concentrators (LSCs). To minimize detrimental reabsorption losses, six semiconductors, typically used to fabricate QDs, with a wide range of the bulk bandgaps $E_{g 0}$ have been considered: $\mathrm{CdS}\left(E_{g 0}=2.42 \mathrm{eV}\right)$, CdSe $\left(E_{g 0}=1.67 \mathrm{eV}\right), \operatorname{CdTe}\left(E_{g 0}=1.5 \mathrm{eV}\right), \operatorname{InP}\left(E_{g 0}=1.27\right.$ $\mathrm{eV})$, InAs $\left(E_{g 0}=0.355 \mathrm{eV}\right)$, and $\mathrm{PbSe}\left(E_{g 0}=0.27 \mathrm{eV}\right)$. We prove that by adjusting the QD radius $\bar{r}$ and dispersion $\Delta \bar{r}$, it is possible to optimize nanocrystal dimensions to minimize the reabsorption. It was shown that for the semiconductor bulk band gap range between $2.42 \mathrm{eV}$ to $1.27 \mathrm{eV}$ there is always the optimum QD size and its dispersion, at which the reabsorption is below the total experimental error of the measured normalized both absorption coefficient and luminescence intensity. Further reduction of $E_{g 0}$ increases the reabsorption at any values of $\bar{r}$ and $\Delta \bar{r}$ : for instance, for PbSe based QD with $E_{g 0}=$ $0.27 \mathrm{eV}, 1 \mathrm{~nm}$ mean radius and its $1 \%$ dispersion, the reabsorption reaches $54 \%$. We estimate the width of the part of the solar spectrum, from which the photons contribute to the luminescence processes. This is important for several LSCs, stalked on top of each other.
\end{abstract}

Key words Photoluminescence, quantum dots, luminophore, reabsorption, solar cells, efficiency, concentrator.

\section{Introduction and the goal}

Luminescent solar concentrators (LSCs) are recently attracting significant attention from both fundamental research and their application as a part of the integrated photovoltaics [1-4]. LSCs can be used, for instance, to build semitransparent photovoltaic windows in urban buildings, thus providing extra energy generation units. Therefore, LSCs can utilize a wide amount of surfaces, which at the moment are neglected, such as windows, skylights, shading devices, etc. LSC typically consists of a waveguide, doped with highly emissive chromophores. The chromophores re-emit the absorbed broad spectra of direct or diffuse sunlight into sufficiently narrow lumi- nescent band at a longer wavelength. The emitted luminescence photons propagate inside the waveguide, formed by LSC slab, through the total internal reflection and are converted into electricity by photovoltaic cells, installed along the slab perimeter, the so-called harvesting edges (Fig. 1). Because the surface area of the slab exposed to sunlight is much larger than the area of its edges, the LSC effectively acts as a solar concentrator. The different types of LSCs can be assembled in a stack to improve the energy harvesting of the wide solar spectrum. There are three main types of chromophores (also called luminophores or phosphors) used in LSCs: (1) rare earth atoms, (2) organic dyes, and (3) quantum dots. As it was demonstrated in [4], the quantum dots are the best suited luminophore compared to the rear earth atoms or dyes. For instance, possessing crystalline semiconductor structure, QDs are stable and less degradable than organic dyes and their spectra can be tuned by modifying of the size. However, the record efficiency $\eta_{c}$ of only $7.1 \%$ has been achieved experimentally for the system of fluorescent concentrators, doped with two dyes [5]. This is substantially below the fundamentally attainable efficiency limit [4] due to several losses, which existing LSCs are suffering from. The dominant losses are due to escape cone and below-unity quantum yield, see Fig. 1.

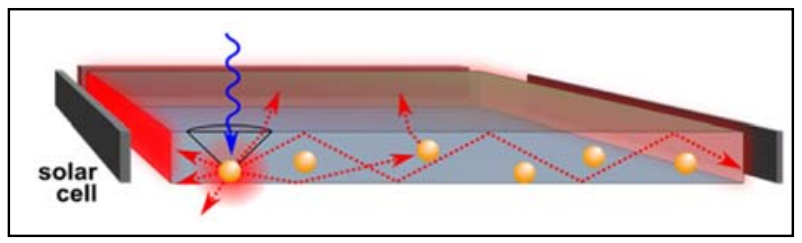

Fig. 1. (Color online.) Perspective view of the luminescent solar concentrator (LSC) (courtesy of [5]). Incoming light photons (blue arrow) are absorbed by luminophore first and then are reemitted. Fluorescent photons, that emit under the angle, smaller then critical angle (black cone at the left), leave the matrix. The solar cells should be spectrally matched to the irradiance of the luminescent material, incorporated into the concentrator. 
However, the above two losses are further aggravated by the reabsorption process, originating due to the overlap of the absorption and emission spectra of the chromophore. They are schematically demonstrated in Fig. 2. The overlap area between the spectra (hatched) is proportional to a vertical position of the crossing point (indicated by a horizontal black arrow). The luminescent photons, emitted within the energy range $\Delta$, can be reabsorbed. The crossing point position is always below 1 and we can use this value in percent as a measure of the reabsorption. As it is shown in [6], the losses due to the reabsorption process can significantly reduce the LSC efficiency. To mitigate these losses, it is necessary to increase a separation $\Delta(h v)$ of the absorption and emission processes in energy. We show below that the losses can be tuned and reduced by properly adjusting QDs gap and geometric characteristics.

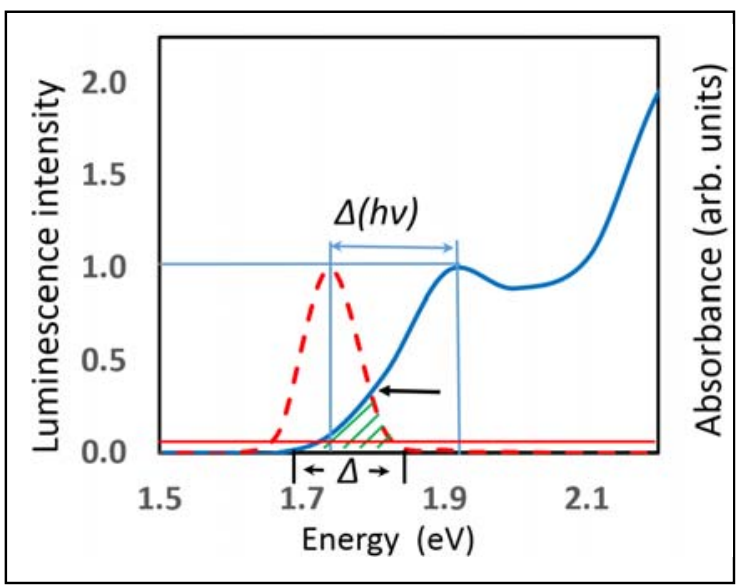

Fig. 2. Overlap of the QD first maximum absorption peak (solid blue line) and luminescence band (red dashes), both normalized to unity. The two spectra overlap between 1.7 and $1.9 \mathrm{eV}$ (hatched). Luminescent photons, emitted in this energy range $\Delta$ can be reabsorbed. The energy separation between the first peak of absorption spectrum and the peak of QD luminescence is $\Delta(h v)$. The horizontal arrow indicates the point where the absorption and luminescence spectra are crossing. The red horizontal line indicates the total accuracy of absorption and luminescence measurements

In the papers, cited above, the correlation between the evolution of the absorption as well as luminescence spectra and the QD size and its dispersion is sufficiently well established and agree well with the experiment. In this work, however, we rather focus on establishing the correlation of QD size and its dispersion with the reabsorption process. We demonstrate that proper choice of the average QD radius $\bar{r}$ and its dispersion $\Delta \bar{r}$ for the bulk band gap ranging between $2.42 \mathrm{eV}$ and $1.27 \mathrm{eV}$, leads to reduction of the overlap of the absorption and luminescence bands, which results in negligible reabsorption losses.

To evaluate the reabsorption losses when using semiconductors with different bulk band gaps and geometrical characteristics of QDs, we start with the established formalisms, used in the literature, see, e.g., [6-8]. The theoretical formalisms, developed in the above references, allow to accurately describe modification of the QD absorption and luminescence spectra for QD ensemble with different size, its dispersion and the bulk band gap of the core semiconductor.

\section{Details of the Calculation and the Results}

We derived the expressions for the QD photon energy dependent both absorption and luminescent emission. They incorporate the Gaussian spreading of the energy levels with the standard deviation $\sigma_{r}$ due to temperature smearing and QDs size dispersion with the standard deviation $\sigma_{E}$. The derived expression for a shape of the luminescence spectrum can be written as:

$I_{P L}\left(E_{p h}\right)=B \int_{0}^{\infty} \frac{1}{r_{i}^{3}} \exp \left(\frac{\left(E_{p h}-E_{P L}\left(r_{i}\right)\right)^{2}}{2 \sigma_{E}^{2}}\right) \exp \left(\frac{\left(r_{i}-\bar{r}\right)^{2}}{2 \sigma_{r}^{2}}\right) d r$,

where $B$ is the constant, independent on the radius, and is determined from the normalization condition of the luminescence intensity $I_{P L}\left(E_{p h}\right)$ to unity. Here $E_{P L}\left(r_{i}\right)$ is the energy of the luminescence intensity maximum of the QD of radius $r_{i}$ with its mean value $\bar{r}, E_{p h}$ is the photon energy. Detailed derivation of the expressions and the calculations will be given elsewhere.

We model QDs as a spherical semiconductor core of the radius $r$ inside the insulating shell, which ensures high luminescence yield. The effects of QD size variation and dispersion on their energy states are analyzed. In the majority of research on the efficiency of photovoltaic solar luminescent concentrators, the polymer plate with QDs is used. Since the plastic plate is transparent in the wavelength range of $0.4-1 \mu \mathrm{m}$, (or $3.1-1.2 \mathrm{eV}$ ), the analysis of the effect of the QD radius and its dispersion on the reabsorption has been performed for the semiconductor QD core with the optical bandgap in the range of $1.2-3.1 \mathrm{eV}$.

Using as an example InP QDs $\left(E_{g 0}=1.27 \mathrm{eV}\right)$, in Fig. 3 we demonstrate how the overlap between the absorption and luminescence spectra depends on the QD size and its dispersion, $10 \%, 5 \%, 2 \%$ and $1 \%$ of QDs dispersion values are considered.

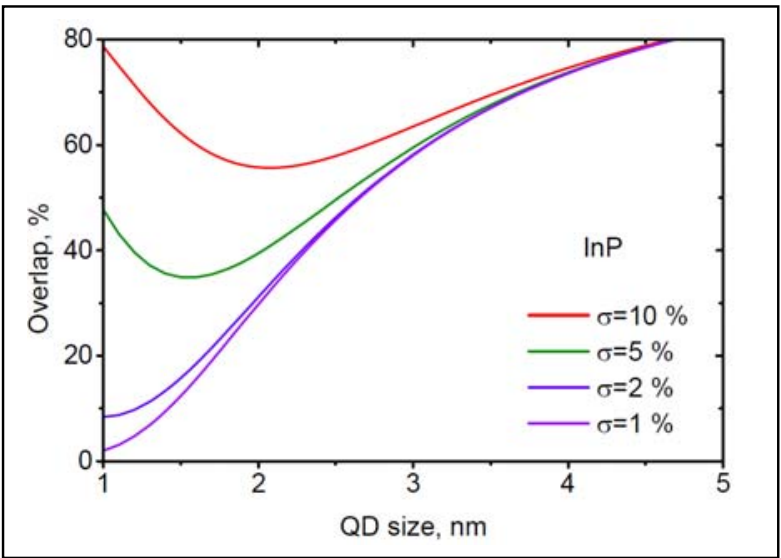

Fig. 3. Dependence of the reabsorption intensity on the QD core radius at four different size dispersions for InP with the gap $E_{g} 0$ $=1.27 \mathrm{eV}$. E.g., if $\sigma=5 \%$, the minimal radius $r_{\min }=1.6 \mathrm{~nm}$.

As a distinct feature of the theoretical curves in Fig. 3, the 
presence of the minimum indicates that the optimum QD core sizes $r_{\min }$, which minimizes the reabsorption, can always be chosen. Indeed, QD size increase above $r_{\text {min }}$ reduces the energy separation between the peaks of the absorption and luminescence, thus increasing the reabsorption. When QD size decreases below $r_{\min }$, the effect of thermal broadening of the quantized energy levels results in the reabsorption growth. Considerable spectra overlap for the QD with the dispersion above 2\% sets the limit for the structural characteristics of the nanocrystal from the point of view of the LSC efficiency.

The results on the reabsorption dependence on the energy gap $E_{g 0}$ for the set of semiconductors chosen is summarized in Fig. 4. It shows that the reabsorption losses are increasing with increase of the QD size dispersion and decrease of the semiconductor bulk gap.

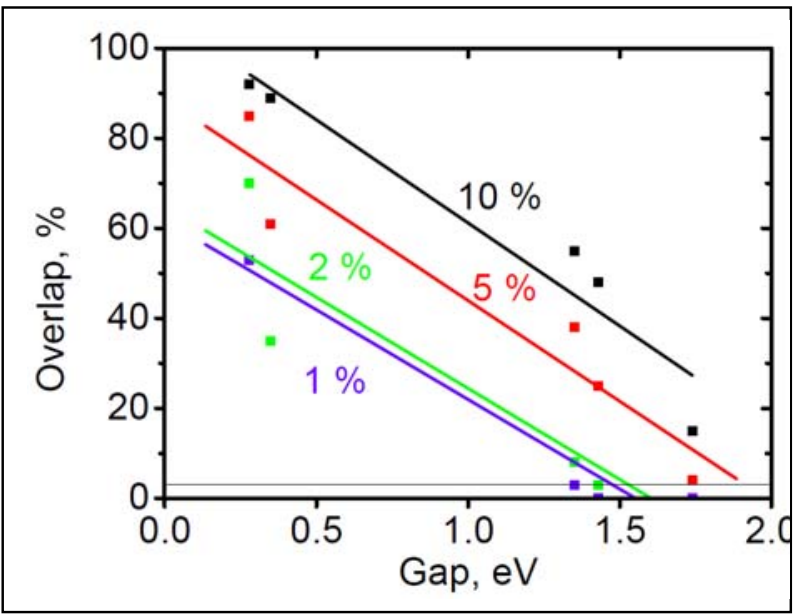

Fig 4. Dependence of the reabsorption losses on the QD optical band gap of the core bulk semiconductor $E_{g 0}$ for different nanoparticle size dispersion. Black horizontal line, close to the $x$ axis, aims to compare the normalized experimental accuracy of the measured QDs spectra to the reabsorption losses.

In particular, the resulting reabsorption dependence on the optical bandgap of QD core, demonstrates that the slope of the reabsorption dependence decreases with reduction of the QD size dispersion. This means that with the $C D$ size dispersion decrease, the sunlight absorption bandwidth also decreases. In particular, for the QD cores with a 5\% size variation, the range of absorption of solar light photons extends the high-side starting from $2.7 \mathrm{eV}$.

\section{Conclusions}

We approached the problem of reduction of the reabsorption losses in the luminescent solar concentrators by analysing how to minimize the overlap between quantum dot absorptions and luminescent bands by modifying the QD radius and its dispersion: QDs are the most promising chromophore in LSC due to a possibility of tuning the absorption and emission spectra by adjusting core diameter. The quantum dots reabsorption characteristics have been investigated considering their dependence on the QD core size, its dispersion and the bulk band gap of the semiconductor core. It is shown that with the core size decrease there exists the optimum QD radius that minimizes the reabsorption. Decreasing or increasing the size of the QD core with respect to the optimal size always increase reabsorption. It was found that with decreasing the bandgap $E_{g 0}$ of the core semiconductor (while $E_{g 0} \geq 1.8$ $\mathrm{eV}$ ) and below or equal to $5 \%$ size dispersion, it is possible that the reabsorption can be negligibly small (that is, below the experimental detection limit). In contrast, when $E_{g 0} \leq 1.8 \mathrm{eV}$, further band gap $E_{g 0}$ decrease only leads to the growth of the reabsorption. For instance, for highly homogeneous with only $1 \%$ size dispersion, $\mathrm{PbSe}$ QDs with $E_{g 0}=0.27 \mathrm{eV}$ the lowest reabsorption of $54 \%$ is still too large.

It was proven that the reabsorption losses can efficiently be minimized in the QDs made from semiconductors with the bulk gap above $1.27 \mathrm{eV}$ and their size dispersion below $2 \%$. The results are important for improving the efficiency of the luminescent solar concentrators.

\section{Acknowledgments}

The authors acknowledge the support from IntriEnergy ${ }^{\mathrm{TM}}$

\section{References}

[1] S. Baskoutas and A. F. Terzis, "Size-dependent band gap of colloidal quantum dots" J. Appl. Phys. Vol. 99, pp. 013708-1 - 013708-4, (2006).

[2] X. Huang, S. Han, W. Huang, and X. Liu, "Enhancing solar cell efficiency: The search for luminescent materials as spectral converters". Chem. Soc. Rev. Vol. 42, pp. $173-$ 201, (2013).

[3] C. Li, W. Chen, D. Wu, D. Quan, Z. Zhou, J. Hao, J. Qin, Y. Li, Z. He, and K. Wang, "Large stokes shift and high efficiency luminescent solar concentrator incorporated with CuInS2/ZnS quantum dots." Scientific Reports Vol. 5, pp. 17777-1 - 1777-9, (2015).

[4] M. R. Kulish, V. P. Kostylyov, A. V. Sachenko, I. O. Sokolovskyi, D. V. Khomenko, and A. I. Shkrebtii, "Luminescent converters of solar light into electrical energy. Review." Semicond. Phys, Quantum Electronics \& Optoelectronics, to be published (2016).

[5] L. R. Bradshaw, K. E. Knowles, S. McDowall, and D. R. Gamelin, "Nanocrystals for luminescent solar concentrators." Nano Letters Vol. 15, 1315 - 1323, (2015).

[6] F. Meinardi, H. McDaniel, F. Carulli, A. Colombo, K. A. Velizhanin, N. S. Makarov, R. Simonutti, V. I. Klimov, and S. Brovelli, "Highly efficient large-area colourless luminescent solar concentrators using heavy-metal-free colloidal quantum dots". Nat. Nano Vol. 10, 878 - 885, (2015).

[7] S. Flügge, "Practical quantum mechanics", Springer, Berlin (1999). P. 155 Problem 62 "Particle enclosed in a sphere." [8] J. Jasieniak, L. Smith, J. van Embden, P. Mulvaney, and M. Califano, "Re-examination of the Size-Dependent Absorption Properties of CdSe Quantum Dots". J. Phys. Chem. C Vol. 113, 19468 - 19474, (2009).

[9] D. Bera, L. Qian, T.-K Tseng and P.H. Holloway, "Quantum Dots and Their Multimodal Applications: A Review" Materials, 3, 2260 - 2345, (2010). 